\title{
La Convención
}

de las Naciones Unidas

sobre la Utilización

de las Comunicaciones

Electrónicas en los Contratos

Internacionales: relevancia

práctica y lecciones

aprendidas $^{*}$

\section{LUCA G. CASTELLANI}

Resumen: La Convención de las Naciones Unidas sobre la Utilización de las Comunicaciones Electrónicas en los Contratos Internacionales es el primer tratado suscrito con el objetivo de facilitar el uso transfronterizo de las comunicaciones electrónicas en las relaciones comerciales. Este artículo describe los objetivos de la política detrás de la preparación de dicho instrumento, su ámbito de aplicación y sus principales disposiciones sustantivas. Se hace referencia específica a la interacción de esa Convención con otros tratados ya adoptados por Colombia y otros Estados, como la Convención sobre el Reconocimiento y Ejecución de las Sentencias Arbitrales Extranjeras (la "Convención de Nueva

* Trad. del inglés: Carlos García Guzmán.

Fecha de recepción: 30 de septiembre de 2015 . Fecha de aceptación: 30 de octubre de 2015. Para citar el artículo: L. G. Castellani, "La Convención de las Naciones Unidas sobre la Utilización de las Comunicaciones Electrónicas en los Contratos Internacionales: relevancia práctica y lecciones aprendidas", Revista de Derecho Privado, Universidad Externado de Colombia, n. ${ }^{\circ}$ 29, julio-diciembre de 20 I 5, pp. 75-99. DOI: http://dx.doi.org/I o. I 860 I/o I $234366 . n 29.04$

** Doctor en Derecho Comparado de la Universidad de Trieste. Magíster en Derecho Internacional de la Universidad de Nueva York. Oficial legal de la Secretaría de la cNUdmi, donde ejerce las funciones de secretario del Grupo de Trabajo Iv (Comercio Electrónico) de la cNudmi, Viena, Austria. Las opiniones expresadas en este documento son del autor y no reflejan necesariamente la opinión de las Naciones Unidas. Contacto: luca.castellani@uncitral.org 
York") y la Convención de las Naciones Unidas sobre Contratos de Compraventa Internacional de Mercaderías (CISG). Por último, el artículo analiza los patrones en la adopción de la Convención en la última década y concluye que Colombia debería considerar la posibilidad de ratificar la Convención, que ha firmado en 2007 , para fortalecer su economía digital mediante la modernización de la legislación vigente sobre el comercio electrónico.

Palabras clave: Convención de las Naciones Unidas sobre la Utilización de las Comunicaciones Electrónicas en los Contratos Internacionales, compraventa internacional de mercaderías, arbitraje internacional, ley de comercio electronico, CNUDMI.

\section{United Nations Convention on the Use of Electronic Communications in International Contracts: Practical relevance and lessons learned}

Aвstract: The United Nations Convention on the Use of Electronic Communications in International Contracts is the first treaty aiming at facilitating cross-border use of electronic communications in commercial relations. The article describes the policy goals behind the preparation of that Convention, its scope of application and its main substantive provisions. It makes specific reference to the interaction of that Convention with other treaties already adopted by Colombia and by numerous other States, such as the Convention on the Recognition and Enforcement of Foreign Arbitral Awards (the "New York Convention") and the United Nations Convention on Contracts for the International Sale of Goods (CISG). Finally, the article analyzes the patterns in the adoption of the Convention in the last decade and concludes that Colombia should consider ratifying the Convention, which it has signed in 2007 , to support its digital economy by modernizing the existing legislation on electronic commerce.

Keywords: United Nations Convention on the Use of Electronic Communications in International Contracts, international sale of goods, international arbitration, Model Law on Electronic Commerce, uncitral.

La Comisión de las Naciones Unidas para el Derecho Mercantil Internacional (CNUDMI, o UNCITRAL por sus siglas en inglés) es el órgano central en el sistema de las Naciones Unidas para la armonización y modernización del derecho comercial internacional. Dicho mandato se desarrolla, entre otras cosas, mediante la preparación de textos legales uniformes y la promoción de su interpretación uniforme. Aunque la CNUDMI usualmente ejerce la función de armonizar leyes 
existentes, esta Comisión también se ha ocupado de temas que no han encontrado aún un adecuado tratamiento legal. Este es el caso en lo que respecta al derecho del comercio electrónico.

De hecho, la cNudmi empezó a trabajar en el tema del comercio electrónico en la década de I980, cuando existían muy pocas leyes al respecto. El trabajo de la CNUDmi llevó a la adopción de dos textos, la Ley Modelo de la cNUdmi sobre Comercio Electrónico ${ }^{\mathrm{I}}$ (MLEC por sus siglas en inglés) y la Ley Modelo de la CNUDMi sobre las Firmas Electrónicas (MLEs por sus siglas en inglés)², habiendo sido ambas significativamente exitosas. Estas leyes modelo son comúnmente consideradas como estándares legislativos globales en sus respectivos campos 3 , y los principios en los que se basan constituyen pilares globales del derecho del comercio electrónico.

Sin embargo, las leyes modelo tienen limitaciones intrínsecas provenientes de su naturaleza de "derecho blando" (Soft law). Por ejemplo, sus disposiciones pueden ser modificadas de diversas maneras una vez son incorporadas en el ordenamiento doméstico. Dichas variaciones inevitablemente afectan la uniformidad $y$, en consecuencia, la previsibilidad legal, de modo especial en lo atinente a las transacciones transfronterizas. Más aún, la MLEC y la MLEs fueron preparadas en un tiempo en el que la predominancia de ciertos modelos tecnológicos, como el intercambio electrónico de datos (EDI), era mayor que el día de hoy, y cuando muchos otros modelos estaban aún por aparecer. De igual manera, nuevos modelos de negocios y prácticas aparecen continuamente. La conveniencia de analizar la experiencia adquirida con la MLEC y la MLEs, así como de la incorporación de disposiciones que atienden a prácticas y tecnologías comerciales recientes, salta a la vista. Finalmente, los requisitos formales incluidos en los tratados pueden constituirse en obstáculos para el reconocimiento legal del uso de las comunicaciones electrónicas. La legislación doméstica, incluso si estuviese basada en una ley uniforme, puede no ser suficiente para hacer frente a aquellos casos que requieren una solución en el mismo nivel del tratado. Sobre la base de

I Ley Modelo de la cNudmi sobre Comercio Electrónico con la Guía para su incorporación al derecho interno, junto con su nuevo artículo 5 bis aprobado en I998, Nueva York, I 999 (publicación de las Naciones Unidas, n. ${ }^{\circ}$ de venta: S.99.V.4). De aquí en adelante, "MLEc". Más de 60 Estados han adoptado la MLEc.

2 Ley Modelo de la cNudmi sobre las Firmas Electrónicas con la Guía para su incorporación al derecho interno, Nueva York, 2002 (publicación de las Naciones Unidas, n. ${ }^{\circ}$ de venta: S.02.V.8). De aquí en adelante, "MLEs". Más de 30 Estados han adoptado la MLEs.

3 Información respecto de la adopción de la MLEC y la MLEs está disponible en el sitio web de la CNUDMI, respectivamente en: http://www.uncitral.org/uncitral/es/uncitral_texts/electronic_commerce/I996Model_status.html y http://www.uncitral.org/uncitral/es/uncitral_texts/ electronic_commerce/200IModel_status.html Dicha información puede encontrarse incompleta debido a que las diferentes jurisdicciones no siempre comunican la adopción de los textos a la Secretaría de la cNUdmi. Por consiguiente, es probable que las jurisdicciones que han adoptado los textos sean más que aquellas que se encuentran enunciadas en el sitio web. 
estas consideraciones, la CNUDMI decidió preparar el primer tratado abordando específicamente el tema del derecho del comercio electrónico. Este trabajo llevó a la adopción de la Convención de las Naciones Unidas sobre la Utilización de las Comunicaciones Electrónicas en los Contratos Internacionales, adoptada por la Asamblea General de las Naciones Unidas el 23 de noviembre de 2005 (E-CC por sus siglas en inglés) ${ }^{4}$. Dada la importancia del tema para el comercio mundial, la E-cC atrajo un amplio e inmediato interés, así como apoyo y elogios por su contenido 5 .

El objetivo principal de la E-Cc es la facilitación del reconocimiento jurídico transfronterizo de las comunicaciones y de las firmas electrónicas. De hecho, el reconocimiento legal transfronterizo y la interoperabilidad técnica son los dos elementos necesarios para permitir transacciones electrónicas a través de las fronteras. Sin embargo, antes de la adopción de la E-CC no existía ningún tratado multilateral específico que pudiera lograr dicho reconocimiento jurídico transfronterizo.

Más específicamente, la E-cc promueve el comercio electrónico transfronterizo al: (I) facilitar el uso de medios electrónicos en relación con la aplicación de tratados concluidos antes de que el uso de las comunicaciones electrónicas fuera común; (2) reforzar los niveles de uniformidad en la promulgación, inter-

4 Colección de tratados de las Naciones Unidas, número de registro I-50525 (publicación pendiente); en adelante, "E-CC". La E-CC fue adoptada por la Resolución 60/2 I de la Asamblea General el 9 de diciembre de 2005 (A/RES/60/2 I).

5 Una bibliografía de los textos de la CNUDmi sobre comercio electrónico, incluida la E-CC, es regularmente compilada por la Secretaría de la cNUDmi y se encuentra disponible en la página web http://www.uncitral.org/uncitral/es/publications/bibliography.html Ver, en particular: A. Boss y W. Kilian (eds.), The United Nations Convention on the Use of Electronic Communications in International Contracts: an in-depth guide and sourcebook, 2008; D. HettenBach, Das Übereinkommen der Vereinten Nationen über die Verwendung elektronischer Mitteilungen bei internationalen Verträgen, 2008 ; K. W. Chong y J. S. CHAo, "United Nations Convention on the Use of Electronic Communications in International Contracts: A new global standard", Singapore Academy of Law Journal, i 8, pp. I I6-202, 2006 (disponible en: http://www.sal.org.sg/digitallibrary/Lists/saL \% 20Journal/Attachments/390/2006- I 8-SAcLJ-I I 6-Chong.pdf); S. EISELEN, "The unecic: International trade in the digital era", Potchefstroom Electronic Law fournal, 2: I-49, 2007 (disponible en: http://www.puk.ac.za/opencms/export/puk/html/fakulteite/regte/per/iss uepages/2007volume Iono2/2007x2x_Eiselen_art.pdf); H. D. GABRIEL, "The United Nations Convention on the Use of Electronic Communications in International Contracts: An overview and analysis", Uniform Law Review / Revue de droit uniforme, vol. I I, n. ${ }^{\circ}$ 2, pp. 285-304, 2006. Entre otros materiales en español: A. Madrid Parra, "El Convenio de Naciones Unidas sobre Contratación Electrónica”, en A. Madrid Parra (dir.), M. J. Guerrero Lebrón (coord.), Derecho patrimonial y tecnología, 2007, pp. 39- I I 3; J. Oviedo Albán, "Convención de las Naciones Unidas sobre la Utilización de Comunicaciones Electrónicas en Contratos Internacionales”, International Law. Revista Colombiana de Derecho Internacional, n. ${ }^{\circ}$ 7, pp. 9-57, 2006 (disponible en: http://revistainternationallaw.javeriana.edu.co/descargas.php?archivo=rev7_cap I. pdf\&idArt=92 \&edicion=7); S. L. Feldstein de Cárdenas y L. B. Scotti, "La Convención sobre Utilización de las Comunicaciones Electrónicas en los Contratos Internacionales: un avance hacia la armonización legislativa en materia de contratación electrónica", Revista Científica de UCES, vol. I I, n. ${ }^{\circ}$ I, pp. 59-89, 2007. 
pretación y aplicación de la MLEC y la MLEs; (3) actualizar y complementar ciertas disposiciones de la MLEC y la MLEs; y (4) proveer una legislación básica moderna y uniforme sobre comercio electrónico a países que carezcan de legislación o que la tengan pero incompleta.

A ese respecto debe notarse que, mientras la E-cc como un tratado tiene naturaleza de "derecho duro" (Hard law), también puede operar como un instrumento de "derecho blando", en una manera similar a una ley modelo. En otras palabras, las disposiciones sustantivas de la E-Cc pueden ser incorporadas en el derecho doméstico, aun cuando la Convención no ha sido formalmente adoptada. Sin embargo, dicho uso de la E-CC no es totalmente recomendable pues no permite alcanzar los beneficios derivados de la E-CC resultantes de su naturaleza como tratado internacional.

Un caso diferente puede darse cuando un Estado, al adoptar la E-cC, decide alinear su legislación nacional con las disposiciones de la E-cc ${ }^{6}$. Esta elección se hace para asegurar que las mismas reglas aplican a las transacciones nacionales e internacionales, evitando de esa manera un régimen jurídico dual y la consiguiente necesidad de determinar la naturaleza nacional o internacional de la transacción. Un beneficio adicional que surge de dicha elección es la consecución de mayor uniformidad legislativa a nivel nacional.

Los textos más influyentes utilizados en la preparación de la E-Cc han sido, por una parte, la Convención de las Naciones Unidas sobre los Contratos de Compraventa Internacional de Mercaderías de I980 (CISG por su siglas en inglés), en lo que respecta al ámbito de aplicación y ciertas cláusulas finales, y, por otra, la MLEC y la MLEs en lo que concierne a las disposiciones sustantivas sobre transacciones electrónicas, incluyendo firma electrónica y contratación electrónica. Este trasfondo es relevante, pues varios precedentes judiciales que dieron aplicación a la CISG, la MLEC y la MLES pueden ser útiles para ilustrar el funcionamiento de disposiciones correspondientes de la E-CC.

\section{Ámbito de aplicación de la E-CC}

El artículo I de la E-cc define su ámbito de aplicación de la siguiente manera: la E-CC "será aplicable al empleo de las comunicaciones electrónicas en relación con la formación o el cumplimiento de un contrato entre partes cuyos establecimientos estén en distintos Estados". Este artículo está inspirado en el artículo I de la CISG, y el carácter internacional de la comunicación electrónica es, al igual

6 Algunos Estados, como Australia y Singapur, han decidido adoptar la E-cc junto a una reforma de la legislación nacional; para una decisión en sentido contrario, ver la legislación canadiense uniforme de implementación de la E-cc: Conferencia de Derecho Uniforme de Canadá, Sección de Derecho Civil, Ley Uniforme de Comunicaciones Electrónicas (Uniform Law Conference of Canada, Civil Law Section, Uniform Electronic Communications Convention Act), aprobada en Winnipeg, Manitoba, agosto de 20 I I. 
que en la CISG, determinado por la ubicación del establecimiento de las partes, como se identifica también a la luz de las normas específicas contenidas en el artículo 6 de la E-cc. Sin embargo, la E-cc, a diferencia del artículo I (I) (a) de la CISG, no requiere que todas las partes implicadas tengan su establecimiento en los Estados contratantes. Por lo tanto, la E-CC se aplica si la ley aplicable a las comunicaciones electrónicas (como se determina en virtud de las normas del derecho internacional privado o, si fuese apropiado, por la lex fori) es la legislación de un Estado Parte de la E-Cc, o si las partes han elegido válidamente la legislación de un Estado Parte de la E-cc como ley aplicable, o han optado por la E-CC en sí misma en los casos en los cuales la elección de disposiciones no estatales se encuentra permitida.

Adicionalmente, la E-CC puede ser aplicada si sus disposiciones sustantivas se encuentran incorporadas en un contrato en virtud del acuerdo entre las partes.

En la práctica, hay que señalar que la Convención puede ser aplicada como un tratado independiente, en relación con una comunicación o un contrato, o en combinación con otro tratado (ver más abajo, art. 20 de la E-Cc). En este último caso, el otro tratado regula el fondo de la cuestión en disputa, y es probable que sea relevante también para determinar la aplicabilidad de la E-cc.

Los bloques sobre los cuales se construye el ámbito de aplicación de la E-cC son las definiciones de "comunicación” y de "electrónico". "Comunicación” se define en el artículo 4 (a) de la E-cc como "exposición, declaración, reclamación, aviso o solicitud, incluida una oferta y la aceptación de una oferta, que las partes hayan de hacer o decidan hacer en relación con la formación o el cumplimiento de un contrato". Esta definición tiene como objetivo incluir tanto intercambios contractuales como los precontractuales. Más aún, la interacción no tiene que realizarse totalmente en forma electrónica, ya que solo algunas partes de ella pueden ser desmaterializadas. Por ejemplo, un documento comercial electrónico podría ser producido en el marco de un contrato de medios mixtos; más aún, este documento comercial podría incluir una cláusula arbitral referida a regulaciones diferentes al derecho sustantivo utilizado en dicho documento.

Por su parte, la definición de "electrónico" se refiere a la definición de "mensaje de datos", que figura en el artículo 4 (c) de la E-CC y se construye sobre la misma definición contenida en la MLEC y la MLEs. "Mensaje de datos" conecta la definición legal de "comunicación electrónica" con la amplia gama de tecnologías disponibles, garantizando así neutralidad tecnológica.

La E-CC se aplica a todas las comunicaciones intercambiadas en relación con la formación o el cumplimiento de un contrato mediante el uso de todos los medios de comunicación que no son escritos u orales ${ }^{7}$. Las excepciones a ese vasto alcance figuran en el artículo 2 de la E-cc, el cual excluye del ámbito de

7 Las comunicaciones escritas y orales adquieren una naturaleza electrónica cuando son grabadas en un medio electrónico. 
aplicación de la Convención: I) los contratos con consumidores definidos en los términos utilizados en el artículo 2 (a) de la CISG; 2) las áreas en las que un alto nivel de uniformidad legal ya ha sido alcanzado por medio de un acuerdo (p. ej., los sistemas de pago internacionales) o de otra manera (p. ej., tratados relativos a valores depositados en un intermediario), y 3) los documentos o títulos electrónicos transferibles que dan derecho a su titular a la entrega de bienes o el pago de una suma como se evidencia en el registro. La razón de esta última exclusión es que normas jurídicas uniformes de carácter amplio para los registros mobiliarios electrónicos están siendo desarrolladas por el Grupo de Trabajo IV (sobre Comercio Electrónico) ${ }^{8}$ de la cNUDmi y por lo tanto no estaban disponibles en el momento de la adopción de la E-cc.

Otros dos elementos pueden variar significativamente el ámbito de aplicación de la E-cc. El primero es el principio general de la autonomía de las partes, el cual apuntala todo el derecho comercial y se materializa en el artículo 3 de la E-Cc, con terminología común a varios de los tratados y leyes modelo de la cNUDMI. Sin embargo, no es deseable una práctica de exclusión voluntaria de la E-cc, ya que esta práctica ha demostrado ser altamente perjudicial en lo que respecta a la CISG $^{9}$.

El segundo es el resultado de las declaraciones de los Estados. De acuerdo con el artículo i9 (I) (a) de la E-cc, un Estado podrá presentar una declaración en el sentido de limitar la aplicación de la E-CC a los casos en que las partes involucradas en la transacción tienen su establecimiento en los Estados contratantes, en virtud de un mecanismo similar al adoptado en el artículo i ( I) (a) de la CISG. Adicionalmente, un Estado puede presentar una declaración en virtud del artículo i9 (I) (b) de la E-cc cuyo efecto consiste en limitar la aplicación de la E-CC a los casos en los cuales las partes así lo elijan: “opt in”. Esta solución, originalmente adoptada en la Convención relativa a una Ley uniforme sobre la formación de los Contratos de Compraventa Internacional de Mercaderías de r 964 (ULF por sus siglas en inglés) ${ }^{\text {Io }}$ y en la Convención relativa a una Ley uniforme sobre la venta internacional de mercancías, I964 (LUCI, ULIS por sus siglas en inglés) ${ }^{\mathrm{I}}$, podría ser útil, por ejemplo, en las jurisdicciones que quieran introducir, gradualmente, nueva legislación. Sin embargo, la decisión de las partes de aplicar la Convención requiere cierto nivel de experiencia en la redacción de contratos, el cual podría no estar presente, en particular, en las pequeñas y medianas empresas, y en los países en desarrollo. Por último, el artículo i 9 (2) de la E-CC da a los Estados la posibilidad de excluir ciertos asuntos del ámbito

8 Para más información, ver el sitio del Grupo Iv de la cNuDMI: http://www.uncitral.org/uncitral/ es/commission/working_groups/4Electronic_Commerce.html

9 Ver L. Spagnolo, CISG Exclusion and Legal Efficiency, 2014.

Io Colección de tratados de las Naciones Unidas, vol. 834, p. I69.

I I Colección de tratados de las Naciones Unidas, vol. 834, p. Io7. 
de aplicación de la E-cc. Estas excepciones podrían ser similares a aquellas que aparecen en la declaración presentada por Singapur y, de forma explícita, por Sri Lanka al ratificar la Convención.

Un asunto interesante es el relacionado con la posibilidad de ampliar el ámbito de aplicación de la E-Cc con respecto a las transacciones electrónicas no comerciales, es decir, a los intercambios entre empresas y consumidores y entre empresas y gobiernos. Esta cuestión se ha planteado en relación con el reconocimiento y ejecución de laudos arbitrales no comerciales (como es el caso de los procedimientos de resolución de disputas en línea que involucran a consumidores) y respecto a permitir un reconocimiento legal mutuo de las instalaciones de ventanilla única para la facilitación del comercio transnacional sin papel. Diferentes soluciones legales pueden estar disponibles para lograr el objetivo previsto: estas radican en presentar una declaración de acuerdo con el artículo 20 de la E-Cc, y en apoyarse en el efecto expansivo del tratado acompañante; por ejemplo, este el caso de la Convención sobre el Reconocimiento y la Ejecución de las Sentencias Arbitrales Extranjeras de I958 ${ }^{[\mathrm{r} 2]}$, en donde dicho efecto radica en su aplicabilidad fuera del contexto puramente comercial.

La legislación regional prevalecerá sobre la E-cc: esa regla general se establece en el artículo I 7 de la E-cc. Este artículo pretende también clarificar la distribución del poder legislativo entre las organizaciones regionales de integración económica y sus Estados miembros. Por lo tanto, las organizaciones de integración económica regional y sus Estados miembros deben depositar una declaración sobre la respectiva competencia legislativa.

\section{Interacción con otros tratados de derecho mercantil internacional}

Uno de los principales objetivos de la E-cc es la eliminación de obstáculos al comercio internacional relacionados con requisitos de tipo formal. En particular, de las referencias a nociones basadas en el uso del papel, tales como "escritura" y "original", establecidas en tratados celebrados antes de la utilización común de medios electrónicos. Por tanto, es útil aclarar que el uso de medios electrónicos funcionalmente equivalentes puede satisfacer esos requisitos de forma. La posición prevalente de los tratados internacionales en el sistema de las fuentes del derecho recomienda establecer una equivalencia al nivel de los tratados internacionales. Para lograr este objetivo, dos enfoques son posibles.

El primer enfoque requiere la modificación formal del tratado con el fin de insertar una referencia a los requisitos de equivalencia funcional, lo cual se hace a través de un protocolo. Posteriormente se realiza la adopción formal del 
texto modificado. Sin embargo, dicho procedimiento modificativo tiene que repetirse para cada tratado, lo cual exige un trabajo legislativo significativo. Adicionalmente, completar los procedimientos de participación en el tratado modificado por parte de todos los Estados parte de la versión no enmendada requiere un período substancial de tiempo; mientras tanto, la existencia de un régimen jurídico dual en la aplicación del tratado puede dar lugar a incertidumbre y disparidad de tratamiento. Finalmente, la decisión de modificar un tratado con el fin de permitir el uso de comunicaciones electrónicas podría entenderse como una negación de la posibilidad de permitir una interpretación liberal en el lenguaje original ${ }^{13}$.

El segundo enfoque tiene por objeto establecer normas generales de equivalencia funcional para las nociones basadas en el uso del papel como "escrito", “firma" y "original”. Este enfoque requiere la adopción de un solo texto que complemente todos los demás instrumentos internacionales pertinentes en relación con las comunicaciones electrónicas en lugar de modificar cada tratado. Esta es la solución adoptada por la E-cC, siguiendo aquellas acogidas en la MLEC y la MLES para establecer la equivalencia funcional en el nivel doméstico. Dos ejemplos pueden ser enunciados para clarificar cómo funciona la E-CC en la práctica.

La Convención de Nueva York de I 958 es el tratado de derecho mercantil internacional más adoptado, actualmente está en vigor en I 56 Estados, y establece requisitos uniformes para el reconocimiento de los acuerdos y laudos arbitrales extranjeros. El artículo in de la Convención de Nueva York ordena el reconocimiento por los Estados parte de los acuerdos arbitrales en forma escrita, y su parágrafo 2 establece que "la expresión 'acuerdo por escrito' denotará una cláusula compromisoria incluida en un contrato o un compromiso, firmados por las partes o contenidos en un canje de cartas o telegramas" ${ }^{4}$. Por otro lado, el artículo iv de la Convención de Nueva York dispone que, con el fin de obtener el reconocimiento y la ejecución del laudo, el solicitante deberá, en el momento de la aplicación, suministrar, entre otros documentos, el acuerdo original al que hace referencia el artículo II, o una copia debidamente certificada del mismo ${ }^{15}$.

I3 Documento de las Naciones Unidas, A/CN.9/485, para. 68. Una interpretación liberal de los requisitos de forma no es inusual, especialmente en las jurisdicciones de common law.

I4 Algunas discrepancias lingüísticas pueden complicar aún más el asunto. Por ejemplo, el uso del término "incluyen" en la versión en inglés del artículo II (2) de la Convención de Nueva York se ha interpretado como una indicación de que la disposición no define exhaustivamente los requisitos de un acuerdo de arbitraje, permitiendo en cambio formas más liberales para cumplir con los requisitos de forma. El texto en las versiones en otros idiomas, sin embargo, indica que la disposición identifica de forma exhaustiva los requisitos necesarios para la validez de un acuerdo de arbitraje.

I 5 El artículo iv de la Convención de Nueva York se refiere también al "original debidamente autenticado de la sentencia o una copia de ese original". La posibilidad de extender la aplicación de la E-cc a los laudos arbitrales puede depender también de la calificación de esos laudos 
Los acuerdos arbitrales celebrados en forma electrónica son cada vez más comunes en la práctica empresarial, dada la progresiva desmaterialización de los documentos comerciales. Por lo tanto, si se busca el reconocimiento y la ejecución de un laudo arbitral de conformidad con la Convención de Nueva York, y el acuerdo de arbitraje se concluyó electrónicamente, es necesario asegurarse de que la ley reconoce la equivalencia entre forma electrónica y en papel. Sin embargo, la E-cc aclara con el máximo nivel de certeza las condiciones en las que el uso de medios electrónicos puede satisfacer el requisito de la forma escrita ${ }^{16}$.

Otro ejemplo de requisitos formales es proporcionado por la CIS $^{17}$. La CISG, adoptada por 83 Estados y ampliamente considerada como un estándar global, ampara la libertad de las partes de configurar el contrato de compraventa de mercancías en lo relativo a los requisitos formales. Sin embargo, los Estados contratantes pueden presentar una reserva, en virtud de los artículos I I, I 2 y 96 de la CISG, exigiendo que los contratos se realicen de forma escrita ${ }^{18}$. En adición a ello, las partes en el contrato de venta de mercaderías podrán acordar la utilización única de comunicaciones escritas sin incluir específicamente el uso de las comunicaciones electrónicas, y podrán exigir el requisito de la firma. Estos acuerdos contractuales pueden ser particularmente relevantes para las modificaciones o enmiendas de un contrato existente (véase el art. 29(2) de la CISG).

Como este se constituye en un requerimiento formal que deroga el régimen liberal de la CISG, es dudoso que la equivalencia entre la forma electrónica y la escrita pueda lograrse a través de la aplicación del derecho interno. La aplicación de la E-cc elimina cualquier duda en este respecto mediante el establecimiento de una equivalencia funcional entre la forma escrita y la forma electrónica.

El mecanismo establecido en la E-CC para la interacción con otros tratados ofrece un abanico de posibilidades. En primer lugar, el artículo 20 de la E-CC enumera varios tratados preparados por la CNUDMI como tratados respecto de

como acuerdos contractuales en virtud de la legislación aplicable (Naciones Unidas, Doc. A/ CN.9/WG.II/WP. I 32 , párr. I I).

I6 La Recomendación relativa a la interpretación del artículo II, párrafo 2, y el artículo viI, párrafo I, de la Convención sobre el Reconocimiento y la Ejecución de las Sentencias Arbitrales Extranjeras, hecha en Nueva York el io de junio de i 958 , adoptada por la cNudmi el 7 de julio de 2006 en su trigésimo noveno período de sesiones (Naciones Unidas, Doc. A/6I/I 7, anexo II), también puede ser relevante. El efecto jurídico de esa declaración en virtud del derecho internacional, sin embargo, se limita a un valor persuasivo o, como mucho, a constituirse en evidencia del creciente consenso internacional.

I 7 Sobre la relación entre la cisg y la E-cc ver Petra Butler, "The cisg and the United Nations Convention on the Use of Electronic Communications in International Contracts", en I. Schwenzer y L. Spagnolo (eds.), State of Play, Eleven International Publishing, Den Haag, 2013, pp. I-i6; S. Eiselen, "The Interaction between the Electronic Communications Convention and the United Nations Convention on the International Sale of Goods", en Boss y Kilian (eds.), ob. cit.

I 8 La declaración de "forma escrita" es una disposición que las partes del contrato no pueden modificar. 
los cuales se establece una equivalencia funcional. Sin embargo, dos tratados preparados por la CNUDMi se excluyen de la lista establecida en el artículo 20, a saber: la Convención de las Naciones Unidas sobre el Transporte Marítimo de Mercancías de 1978 (las "Reglas de Hamburgo") ${ }^{19}$ y la Convención de las Naciones Unidas sobre Letras de Cambio Internacionales y Pagarés Internacionales de $1988^{[20]}$. La razón para dicha exclusión radica en que esos dos tratados contienen disposiciones sobre documentos transferibles, los cuales se encuentran excluidos del ámbito de aplicación de la E-cc.

El Convenio de las Naciones Unidas sobre el Contrato de Transporte Internacional de Mercancías Total o Parcialmente Marítimo de 2008 (las "Reglas de Rotterdam") ${ }^{21}$ también contiene disposiciones relativas a los documentos negociables. No se excluye explícitamente de la E-cc, ya que se concluyó después de la aprobación de esta. Sin embargo, las Reglas de Rotterdam y la E-CC podrían interactuar. Por ejemplo, el artículo 38(2) de las Reglas de Rotterdam exige que los documentos electrónicos de transporte sean firmados, pero no aborda el tema del reconocimiento transfronterizo de las firmas electrónicas.

El artículo 20(2) de la E-CC indica que la E-CC se aplicará a todos los demás tratados de derecho comerciales que contengan requisitos formales, a menos que un Estado declare no estar obligado por dicha disposición. Si esta reserva fuese presentada, en virtud del artículo $20(3)$ de la E-CC el Estado declarante puede identificar ciertos tratados en los que se aplicará la E-Cc. El artículo 20(4) de la E-CC ofrece la posibilidad de excluir un tratado específico de la aplicación de la E-CC. En otras palabras, de acuerdo con el artículo 20(3) de la E-CC un Estado puede optar por excluir de manera general la interacción entre la E-CC y otros tratados, y luego optar por incluirlos de manera selectiva; mientras que de acuerdo con el artículo 20(4) de la E-CC un Estado opta por inclusiones generales, y luego opta de manera selectiva.

Por último, cabe señalar que, incluso si un Estado decidiera excluir toda interacción entre la E-CC y otros tratados, la E-CC mantendría una relevancia fundamental con respecto al establecimiento del régimen jurídico de las comunicaciones electrónicas transfronterizas intercambiadas para propósitos comerciales.

\section{Principales disposiciones sustantivas de la E-CC}

El artículo 5 de la E-Cc establece principios de interpretación utilizando el mismo lenguaje conocido y utilizado, por ejemplo, en el artículo 7 de la cisG, que ha recibido mucha atención en la jurisprudencia y en la doctrina.

I9 Colección de tratados de las Naciones Unidas, vol. I695, p. 3.

20 United Nations Sales Publication n. ${ }^{\circ}$ E.95.V.I6 (el tratado no ha entrado en vigor).

2 I United Nations Sales Publication n. ${ }^{\circ}$ E.og.V.g (el tratado no ha entrado en vigor). 
El artículo 5(I) de la E-cc impone a los jueces una obligación, común a varios textos de la CNUDMI, de interpretar y aplicar la E-CC de manera uniforme. Sin embargo, una diferencia podría presentarse: los textos cuyo objetivo es la armonización de las tradiciones jurídicas existentes, como la cisG, tienen que hacer frente a la "tendencia de regreso a casa" (bomeward trend) ${ }^{22}$, es decir, la tendencia a utilizar, en la interpretación de textos uniformes, nociones nacionales. En el caso de las comunicaciones electrónicas, un estándar legislativo nacional puede no existir o puede no estar bien establecido: por lo tanto, el peligro de una tendencia de "regreso a casa" puede ser menos prominente. No obstante, es importante destacar el carácter autónomo de la E-CC y promover su interpretación uniforme, así como también la de las regulaciones nacionales creadas a partir de las leyes modelo uniformes que complementan la E-cC en el nivel nacional.

El artículo 5(2) de la E-CC se refiere a los principios generales en los cuales se soporta la E-CC como aquellos a utilizarse para resolver cuestiones regidas por la E-cc pero que son específicamente tratadas por ella. Una vez más, esta es una disposición común en los textos de la CNUDMI, cuyo contenido puede variar con cada texto. Algunos principios generales que sustentan la E-CC pueden ser iguales a los identificados con respecto al artículo 7 de la cisg (p. ej., la buena fe y el favor contractus). Otros principios adicionales se pueden encontrar en textos de la CNUDMI sobre las comunicaciones electrónicas, específicamente, la MLEC y la MLEs.

El artículo 6 de la E-CC se refiere el tema de la ubicación de las partes. La noción de "relación más estrecha" contenida en el artículo 6(2) de la E-CC se inspira en el artículo io de la cisg. Sin embargo, una regla específica se introdujo en el artículo 6(I) de la E-cc para ayudar a determinar la ubicación física de una de las partes. De hecho, una característica de la utilización de medios electrónicos es la desmaterialización de la ubicación física; por lo tanto, cada parte tiene que determinar su ubicación. En este sentido, el artículo 6(r) de la E-CC aclara que la autodeterminación del establecimiento puede ser refutada.

Adicionalmente, el artículo 6(4) de la E-cc especifica que la ubicación de los equipos y de tecnología de apoyo o el lugar desde donde se accede a un sistema de información no son necesariamente relevantes para la determinación del establecimiento de una de las partes. Dicha regla puede ser particularmente útil en el caso de utilización de servicios en la nube, caso en el cual es difícil determinar la ubicación de ciertos elementos en el sistema de información. El artículo 6(5) de la E-CC introduce una regla similar con respecto a la utilización de nombres de dominio o direcciones de correo electrónico específicos de un país; ya que la movilidad y portabilidad han hecho que el acceso a las comunicaciones electrónicas sea omnipresente, es posible que dichos elementos no resulten signi- 
ficativos para la determinación del establecimiento de una de las parte. Sobre la base de los mismos principios se puede inferir que la determinación de un establecimiento es diferente a la geo-localización de un sistema de información.

El artículo 7 de la E-cc tiene por objeto garantizar que los requisitos de información establecidos en otros textos no se ven afectados por la aplicación de la E-cc. Estos requisitos pueden ser nacionales o internacionales, de aplicación obligatoria, o adoptados de manera voluntaria.

El artículo 8 de la E-Cc establece a nivel internacional el principio de no discriminación de las comunicaciones electrónicas, formulado en principio en el artículo 5 de la MLEC. La información electrónica meramente incorporada por vía de referencia (p. ej., vinculada o adjunta) tampoco puede ser discriminada por el solo hecho de producirse su incorporación por vía de referencia. Esta regla no es explícita en el artículo 8 de la E-CC, pero puede ser inferida de los principios generales del derecho uniforme de las comunicaciones electrónicas reiterados en el artículo 5 bis de la MLEC. El principio de no discriminación tiene otras implicaciones. Por ejemplo, previene la imposición de ciertos requisitos en relación con el uso de las comunicaciones electrónicas cuando dichos requerimientos no existan en relación a transacciones equivalentes basadas en el uso del papel.

El artículo 8(2) de la E-cc determina que el uso de las comunicaciones electrónicas es opcional, pero aclara también que el consentimiento de su uso puede ser implícito. Iniciar un intercambio electrónico, o simplemente proporcionar una dirección electrónica, podría ser suficiente para transmitir dicho consentimiento implícito. En ambos casos, esto también puede equivaler a una designación implícita de la dirección electrónica relevante en virtud del artículo io de la E-cc.

El artículo 9 de la E-CC es una disposición fundamental que establece la equivalencia funcional entre las comunicaciones electrónicas y las comunicaciones escritas con relación a conceptos clave tales como "escrito", "firma" y "original". Es de fundamental importancia que el derecho asegure de un lado uniformidad transfronteriza en su aplicación y de otro lado flexibilidad para respaldar las prácticas comerciales y los acuerdos contractuales. De hecho, establecer requisitos demasiado rígidos para la equivalencia funcional de "original”, incluyendo la exigencia implícita o explícita del uso de ciertas tecnologías, representaría un obstáculo insuperable para el reconocimiento transfronterizo de las comunicaciones electrónicas. También hay que señalar que estas disposiciones tienen el propósito de operar con respecto a los intercambios electrónicos transfronterizos, mientras que los requisitos para la equivalencia funcional en las transacciones nacionales se establecen en la legislación nacional. Sin embargo, la adopción de la E-CC asegura que las posibles variaciones en la aplicación de la equivalencia funcional a nivel nacional no impidan el reconocimiento legal mutuo de las transacciones electrónicas en el ámbito internacional.

El artículo 9(I) de la E-CC aclara que los requisitos de forma, cuya equivalencia funcional es buscada, deben ser contenidos en otra ley o en los acuerdos contractuales ya que la E-CC no introduce nuevos requisitos de forma. 
El artículo 9(2) de la E-CC establece los requisitos para la equivalencia funcional de la noción de "forma escrita" de la misma manera que el artículo 6(I) de la MLEC: ya que la función de la escritura es proporcionar evidencia futura de la información, dicha función se cumple si la información contenida en la comunicación electrónica está disponible para referencia futura. Las partes deben prestar especial atención para evitar la falta de accesibilidad de la comunicación electrónica debido a la obsolescencia tecnológica.

El artículo 9(3) de la E-cc trata el tema del equivalente funcional de la firma manuscrita. Una firma electrónica satisfará el requisito formal de la firma manuscrita: “(a) Si se utiliza un método para determinar la identidad de esa parte y para indicar la voluntad que tiene tal parte respecto de la información consignada en la comunicación electrónica”, y “(b) Si el método empleado: i) O bien es tan fiable como sea apropiado para los fines para los que se generó o transmitió la comunicación electrónica, atendidas todas las circunstancias del caso, inclusive todo acuerdo aplicable; o ii) Se ha demostrado en la práctica que, por sí solo o con el respaldo de otras pruebas, dicho método ha cumplido las funciones enunciadas en el apartado a) supra".

$\mathrm{El}$ artículo 9(3) de la E-CC innova de manera significativa frente el artículo 7 de la MLEc y el artículo 6(I) de la MLEs. Al no repetir el enfoque de "dos niveles" (two tier) contenido en el artículo 6(3) de la $\mathrm{MLES}^{23}$, el artículo 9(3) de la E-CC promueve la neutralidad tecnológica en su formulación más rigurosa. Más aún, el principio de equivalencia sustancial en el reconocimiento transfronterizo de las firmas electrónicas incluido en el artículo i 2 de la MLEs se encuentra plenamente implementado en el artículo 9(3) de la E-cc. Este principio indica que una firma electrónica extranjera no puede ser discriminada por razón de su origen, pero debe ser evaluada en virtud de los mismos requisitos de fiabilidad utilizados para evaluar una firma electrónica creada en la jurisdicción donde debe ser reconocida ${ }^{24}$.

Adicionalmente, el artículo 9(3) de la E-cc sustituye la noción de "la aprobación de la persona" contenida en el artículo 7 de la MLEc y en el artículo 6(I) de la MLEs con la de "la voluntad de la parte" a fin de captar de mejor manera las distintas funciones asociadas a las firmas. De hecho, las firmas en papel no siempre expresan la aprobación del documento firmado pero pueden satisfacer otras funciones tales como, por ejemplo, dar por entendido el contenido del

23 Bajo el enfoque de "dos niveles", cualquier firma electrónica puede tener reconocimiento legal a la luz de las circunstancias, pero algunas firmas electrónicas, que cumplan con requisitos más estrictos expresados de una manera tecnológicamente neutral, se pueden beneficiar de las presunciones relativas a su origen, integridad, etc.

24 Sobre el principio de equivalencia sustancial en el reconocimiento transfronterizo de las firmas electrónicas, véase: uncitral, Promoting confidence in electronic commerce: Legal issues on international use of electronic authentication and signature methods, Viena, 2009 (publicación de las Naciones Unidas, n. ${ }^{\circ}$ de venta E.o9.V.4), paras. I 58-г 6 I. 
documento, o para atestarlo. El contenido real de la intención asociada a la firma electrónica debe determinarse caso a caso y con base en los términos en que se expresa dicha intención y en todas las demás circunstancias pertinentes. $\mathrm{Al}$ respecto la E-CC exige que el método utilizado claramente indique tanto la expresión de la propia intención ${ }^{25}$ como el consentimiento a someterse a dicha intención ${ }^{26}{ }_{2}^{27}$. Adicionalmente, al enfocarse en los atributos de un método (a diferencia de la noción de datos), el artículo 9(3)(a) de la E-cc amplía la gama de procesos y tecnologías disponibles ${ }^{28}$. Esto podría ser particularmente útil cuando se utilizan tecnologías que fusionan medios electrónicos y no electrónicos.

Una cláusula de seguridad se introdujo en el artículo 9(3)(b)(ii) de la E-CC para asegurar que cuando sea posible identificar al firmante de una comunicación electrónica y su intención con respecto a la comunicación firmada, dicho signatario no pueda repudiar la firma sobre la base del método o la naturaleza de la firma. Sin esta disposición, una comunicación que sería válida de otra forma podría ser invalidada si el método de la firma no fuera tan fiable como sería apropiado, permitiendo posiblemente, de esa manera, que el signatario se eximiera de sus obligaciones contractuales ${ }^{29}$. Este test se conoce como "fiabilidad en la práctica", mientras que el test contenido en el artículo 9(3)(b)(i) de la E-CC se denomina "fiabilidad en la teoría" 3 .

La importancia del artículo 9(3) de la E-Cc es resaltada en razón de la experiencia del artículo i 2 de la MLEs sobre el reconocimiento transfronterizo de las firmas electrónicas. El artículo i 2 de la mLes es una disposición que rara vez se ha adoptado en las legislaciones nacionales pues ha prevalecido un enfoque más rígido en el reconocimiento de las firmas electrónicas extranjeras. Por el contrario, las leyes nacionales a menudo requieren un acto formal de recono-

Por ejemplo, mediante una declaración como: "Firmo con el propósito de expresar esta intención”.

26 Mediante una declaración como: "Mediante la realización de esta acción, soy consciente de que estoy firmando".

27 T. J. Smedinghoff, "Article 9: Form requirements", en Boss y Kilian (eds.), ob. cit., i 53.

28 Ibíd., I48-i 5 I; Chong y Chao, ob. cit., pp. г65-г66.

29 Por ejemplo, en SM Integrated Transware Pte Ltd. v. Schenker Singapore (Pte) Ltd., decisión de la Corte Suprema de Singapur n. ${ }^{\circ} 594$ de 2003, [2005] SGHC 58, Caso clout C 66 i, una de las partes trató de invalidar un contrato celebrado mediante el intercambio de correos electrónicos después de una negociación llevada a cabo por correo electrónico, teléfono y en persona, sobre la base de que los correos electrónicos no cumplirían el requisito de forma escrita exigido por la legislación de Singapur relevante para el contrato (contrato de arrendamiento de tierra), incluyendo la firma obligatoria de la persona contra la que se solicitó el cumplimiento del contrato. Los correos electrónicos no se firmaron y no contenían el nombre escrito del autor en el cuerpo del mensaje. El juez consideró que el requisito de la firma había sido satisfecho por la presencia de la cabecera del mensaje indicando el autor de los mensajes de correo electrónico (campo [De parte de: Nombre del remitente]), un elemento suficiente para identificar al autor del mensaje, junto con otros factores. 
cimiento de una firma electrónica extranjera el cual se produce en virtud de un acuerdo de reciprocidad general entre jurisdicciones, de un acuerdo de certificación cruzada entre un prestador de servicios de certificación extranjera y un proveedor acreditado en el país en el que la firma necesita ser reconocida, o de una combinación de los $\operatorname{dos}^{3}{ }^{\mathrm{I}}$. En la práctica, estos mecanismos han demostrado ser engorrosos y encuentran una aplicación práctica limitada. Por otra parte, con fundamento en los principios de neutralidad tecnológica y equivalencia funcional, el artículo 9(3) de la E-cc adopta una versión versátil del principio de equivalencia sustancial sobre la base de una serie de elementos, incluyendo los factores legales, tecnológicos y comerciales, así como los acuerdos contractuales. Por lo tanto, la E-cc facilita tanto el reconocimiento mutuo de las firmas electrónicas como su validación en caso de litigio ${ }^{32}$.

La importancia del artículo 9(3) de la E-cC para el futuro desarrollo del comercio electrónico transfronterizo no puede ser subvalorada. Los acuerdos bilaterales y multilaterales de libre comercio a menudo contienen una cláusula que obliga al reconocimiento transfronterizo de las firmas electrónicas basadas en estándares tecnológicamente neutrales ${ }^{33}$. Estas disposiciones tienen por objeto promover el comercio electrónico y, en particular, el comercio no basado en el uso del papel. Su aplicación efectiva en el nivel multilateral puede tener lugar solamente a través del mecanismo previsto en el artículo 9(3) de la E-cc.

El artículo 9(4) y (5) de la E-CC contiene los requisitos para establecer el equivalente funcional de la noción basada en papel de "original". Estas disposiciones se inspiran en el artículo 8(I) y (3) de la MLEc.

Las disposiciones sustantivas restantes de la E-CC se refieren a la contratación electrónica. El artículo ıo de la E-cc trata sobre el tiempo y lugar de envío y recepción de las comunicaciones electrónicas y actualiza el artículo i 5 de la MLEC, teniendo en cuenta nuevas prácticas de negocios y la innovación tecnológica.

Según el artículo Io(I) de la E-cc, el envío se produce cuando la comunicación electrónica deja el sistema de información bajo el control del iniciador, mientras que de acuerdo con el artículo i 5 de la MLEC el mensaje de datos 34

3 I Por ejemplo, véase Colombia, Ley 527 de I999, art. 43; Omán, Ley de Transacciones Electrónicas (2008), art. 42(3); véase también la Regulación (EU) n. ${ }^{\circ}$ 910/20 I 4 del Parlamento Europeo y del Consejo sobre servicios de identificación y confianza electrónicos para las transacciones electrónicas en el mercado interior (eIDAs), art. I4.

32 Para una discusión de los factores pertinentes, véase Naciones Unidas, Nota explicativa de la Secretaría de la CNUDMI sobre la Convención de las Naciones Unidas sobre la Utilización de las Comunicaciones Electrónicas en los Contratos Internacionales, Nueva York, 2007 (publicación de las Nciones Unidas, n. ${ }^{\circ}$ de venta E.07.V.2), para. I62.

33 Véase, por ejemplo, el tratado de libre comercio entre Estados Unidos y Corea (KorUs FTA), art. I 5.4, y el tratado de libre comercio entre Estados Unidos y Colombia, art. I 5.6.

34 La noción de "mensaje de datos" en la MLEC corresponde funcionalmente a la de "comunicación electrónica” en la E-cc. 
se distribuye cuando entra en un sistema fuera del control del iniciador ${ }^{35}$. La norma ha sido revisada con el fin de evitar consecuencias para el autor cuando el mensaje no puede entrar en el sistema de información por razones fuera de su control. Esas razones incluyen el uso de un servidor de seguridad y de filtros de spam, y la posibilidad de que los sistemas del destinatario o el intermediario no se encuentren en funcionamiento.

El artículo ro(2) de la E-cc distingue, para la determinación del momento de la recepción de una comunicación electrónica, entre dirección electrónica designada y no designada. Este enfoque ya había sido adoptado en el artículo i 5 (2) de la MLEc. Sin embargo, el artículo io(2) de la E-cc contiene un elemento nuevo con respecto al momento de la recepción de las comunicaciones electrónicas enviadas a una dirección electrónica no designada: en ese caso, la comunicación se considerará recibida cuando es susceptible de ser recuperada y el destinatario es consciente de que se ha enviado la comunicación ${ }^{36}$. Esta regla sigue más de cerca aquella que prevalece en el mundo del papel. Por otra parte, el artículo I 5(2) de la MLEc requiere la recuperación real por parte del destinatario, lo que podría permitir que el destinatario retrase o evite la entrega de un mensaje electrónico al no acceder a él de forma deliberada ${ }^{37}$.

El artículo Io(3) de la E-Cc determina el lugar de envío y recepción de las comunicaciones electrónicas de manera similar al artículo I 5(4) de la MLEc. El artículo Io(4) de la E-cc, complementado por el artículo 6 de la E-cc, proporciona normas complementarias sobre la determinación del lugar de recepción.

El artículo i I de la E-cc establece que una propuesta contractual contenida en una comunicación electrónica y no dirigida a partes específicas, pero que es, en general, accesible a las partes haciendo uso de sistemas de información, se considera una invitación a ofertar, y no una oferta vinculante, a menos que la intención de quedar obligado en caso de aceptación se indique claramente en la propuesta. Esta disposición, inspirada en el artículo I4(2) de la CISG, no existía en los textos anteriores de la CNUDMI sobre comercio electrónico y encuentra su razón de ser en el hecho de que un gran número de comunicaciones electrónicas se puede intercambiar instantáneamente. Por lo tanto, los comerciantes pueden recibir en un corto período de tiempo numerosas aceptaciones vinculantes de

35 Para un caso interesante sobre esta punto, véase Corte Laboral de Sudafrica (Durban), Caso n. ${ }^{\circ} \mathrm{D}_{204 / 07}$ (r. ${ }^{\circ}$ de julio de 2008) Fafta v. Ezemvelo KZN Wildlife [2008] zALC 84; [2008] IO BLLR 954 (LC); (2009) 30 ILJ I3 I (LC); Caso clout Case 964, disponible en: http://www.saflii.org/ $\mathrm{za} / \mathrm{cases} / \mathrm{zALC} / 2008 / 84 \cdot \mathrm{html}$

36 Para una decisión judicial relevante, véase Cour d'appel du Québec (Canada), Services financiers Paccar ltée c. Kingsway, compagnie d'assurances générales, 3 I de mayo de 20I2; 2012 QCCA IO3O (CanliI); Caso clout I I95, disponible en: http://canlii.ca/t/frmgm

37 La misma regla se utiliza en los Principios unidroit sobre los contratos comerciales internacionales (2010), art. I.10.3. El comentario a ese artículo hace referencia explícita al artículo IO (2) de la E-CC. 
sus ofertas en línea. Esta posibilidad requeriría mantener grandes existencias físicas de los bienes transables, lo que lleva a una gestión ineficiente del almacenamiento, o, alternativamente, forzaría a concluir costosas compras para cubrir los déficits. En ambos casos, esto conduciría a un incremento de los precios de los productos para los compradores finales. Por lo tanto, se consideró apropiado dar el control al vendedor sobre la celebración del contrato.

El artículo I 2 de la E-cc especifica que un contrato concluido mediante el uso de sistemas automatizados de mensajes es válido y exigible a pesar de que ninguna persona física haya revisado o intervenido en las acciones llevadas a cabo por el sistema automatizado de mensajes. Esto representa una aplicación del principio de no discriminación con respecto a la utilización de los sistemas electrónicos automatizados ${ }^{3}$.

El artículo I 3 de la E-CC indica que la E-CC no afecta a los deberes de información que a menudo están contenidos en la legislación nacional. La disposición podría ser particularmente relevante si la aplicación de la E-cc se extiende, mediante declaración o acuerdo de las partes, a las transacciones con los consumidores. El preámbulo de la MLEC contiene una observación similar en relación a su alcance que indica que "La presente ley no deroga ninguna norma jurídica destinada a la protección del consumidor".

Un conjunto diferente de consideraciones se refiere al hecho de que dichos deberes de información pueden, en realidad, obstaculizar el comercio electrónico transfronterizo y violar el principio de no discriminación del comercio electrónico, si son requeridos únicamente en relación con el uso de las comunicaciones electrónicas, pero no para el comercio tradicional. Ese último caso no es inusual en materia de protección del consumidor.

El artículo I4 de la E-CC contiene una norma destinada a proteger a las personas naturales de los errores de digitación. Esta disposición no existía en textos anteriores de la CNUDMI y representa una excepción al principio de que los textos de la CNUDMI sobre comercio electrónico no interfieren con el derecho sustantivo. Su justificación está relacionada con las características peculiares de la interacción entre humanos y máquinas. Sin embargo, las repercusiones prácticas del artículo I4 de la E-CC pueden ser limitadas, ya que se podrá retirar un error de digitación solo si se cumplen estas condiciones bastante estrictas: a) el error de digitación se hace por una persona física tratando con un sistema automatizado de mensajes; b) el sistema automatizado de mensajes no proporciona la oportunidad de corregir el error; c) la parte que cometió el error notifica lo

38 Véase, por ejemplo, la interesante discusión sobre el uso de sistemas electrónicos automatizados vis-à-vis el derecho contractual islámico en M. Elsan y M. Subaty, "Contract formation using automated message system: Survey of Islamic contract law", Arab Law Quarterly, vol. 23, n. 2, pp. I67-180, 2009. 
antes posible a la otra parte del error, y d) la parte que cometió el error no ha recibido ningún beneficio por razón de la transacción.

Por lo tanto, si el sistema automatizado de mensajes proporciona al ser humano la posibilidad de revisar y confirmar la información introducida, como es a menudo el caso de las aplicaciones comerciales, el artículo i 4 de la E-cc no se aplica. Por otra parte, en el caso de entrega inmediata del bien o servicio (p. ej., digitalmente) puede ser difícil, si no imposible, que la parte que invoca el error pueda probar que no recibió ningún beneficio de la transacción.

No obstante, el artículo I 4 de la E-Cc puede tener una influencia significativa en el fomento de la adopción de mecanismos para la revisión de las transacciones electrónicas por parte de personas físicas, lo que reduce los errores de digitación.

\section{Tendencias en la adopción de la E-CC}

La MLEC y la MLES representan un notable éxito. Los Estados continúan adoptando esas leyes modelo a un ritmo regular, beneficiándose de este modo no solo de la disponibilidad de una legislación moderna y completa, sino también de un fácil acceso a los estudios académicos y a una creciente jurisprudencia. Estas son ventajas importantes, especialmente para los países en desarrollo, al elaborar y aplicar la nueva legislación. En algunas regiones, los textos de la CNUDMI se consideran normas legislativas comunes de facto 39 . En algunos casos, la MLEC y la MLES se han actualizado con las disposiciones sustantivas de la E-CC cuando se usan para preparar los textos regionales $4^{\circ}$.

La creciente importancia de la dimensión transfronteriza del comercio electrónico recomienda la adopción de la E-cC en conjunción con la promulgación de la MLEC y de la MLEs. No hacerlo priva al marco legislativo del comercio electrónico de la importante dimensión internacional. Por otra parte, la adopción de la E-CC mejora la eficiencia de aquellas jurisdicciones donde la MLEC y la MLEs ya han sido adoptadas al introducir nuevas disposiciones y aclarar el funcionamiento de otras a la luz de las prácticas comerciales actuales y de los últimos avances tecnológicos.

39 Para Asia Sudoriental, véase C. Connelly, "Using the Electronic Communications Convention to harmonize national and international electronic commerce laws: An ASEAN case study", en Boss y Kilian (eds.), ob. cit., pp. 3 I 5-332; UNCTAD, Review of e-commerce legislation harmonization in the Association of Southeast Asian Nations, Nueva York y Ginebra, 20 I3, United Nations Doc. unCtad/Dth/stict/20I3/I. Para América Central y América Latina, véase unctad/Cnuced, Estudio sobre las perspectivas de la armonización de la ciberlegislación en Centroamérica y el Caribe, Nueva York y Ginebra, 20 Io, United Nations Doc. unCtad/DTL/STICT/2009/3; UnCTAD/CNUCED, Estudio sobre las perspectivas de la armonización de la ciberlegislación en América Latina, 2009.

40 Este es el caso del Caribe, con el proyecto de la UIT HIPCAR, y en África Oriental y Meridional, con la Ley Modelo de comesa en Transacciones Electrónicas y Guía para la incorporación de 20 I (COMESA Model Law on Electronic Transactions and Guide to Enactment 20 Io), y con el marco jurídico de la Comunidad del África Oriental para la ciberlegislación. 
Transcurrida la primera década de vigencia de la E-Cc es posible trazar un primer balance respecto de su adopción y aplicación. El número oficial de Estados parte no es particularmente alto: siete Estados la han adoptado formalmente ${ }^{4}$, aunque otros están en una fase avanzada en los procedimientos de adopción ${ }^{42}$. Trece países más firmaron la E-cc pero todavía no la han ratificado, y Colombia se encuentra entre estos. Sin embargo, hay que subrayar que la E-CC es uno de los tratados de la CNUDMi de mayor éxito en los últimos años.

La lenta tendencia hacia la adopción del instrumento es, al menos en parte, debida a los problemas generales que afectan a la aprobación de tratados de derecho mercantil uniforme. Por ejemplo, el tiempo de los parlamentarios es cada vez más escaso debido a la multiplicidad de temáticas que abordan, y las cuestiones de derecho uniforme rara vez son visibles para el público en general, por lo que es poco probable que reciban atención política.

Por otra parte, las jurisdicciones que lideran el proceso de reforma de la legislación comercial se encuentran tradicionalmente ubicadas en los países desarrollados europeos y norteamericanos. Sin embargo, por una parte, los Estados europeos están completamente inmersos en el proceso de integración económica regional, el cual no siempre tiene en cuenta la necesidad de garantizar la interacción con los textos globales uniformes; por otra parte, en las últimas décadas los Estados Unidos de América han sido renuentes a adoptar tratados multilaterales, con independencia de su objeto. Como resultado, el efecto de remolque proporcionado por la adopción de textos uniformes por parte de dichas jurisdicciones se ha perdido. En los países en desarrollo la reforma del derecho comercial no suele ser una prioridad debido a la escasa capacidad legal local, al poco interés de las organizaciones internacionales y otras entidades que puedan tener influencia en la definición de políticas, y al conocimiento limitado de los beneficios asociados. Pero, cuando el asunto logra obtener la atención de los responsables políticos, los tratados son, de hecho, adoptados.

En el caso de la E-Cc, los desafíos para la adopción formal de tratados han contribuido a una situación peculiar en que los Estados reconocen la importancia de las disposiciones de la E-Cc, pero solo las adoptan domésticamente. Esto sucedió en al menos quince jurisdicciones desde Guatemala ${ }^{43}$ hasta Vietnam ${ }^{44}$.

4I El Congo, República Dominicana, Honduras, Montenegro, la Federación Rusa, Singapur y Sri Lanka.

42 P. ej., Australia.

43 Decreto 47-2008 del ig de agosto 2008, "Ley para el reconocimiento de las comunicaciones y firmas electrónicas". El decreto ya cumple con las disposiciones de la E-cc.

44 Decreto 57/2006/ND-CP del 9 de junio de 2006, "Decreto sobre Comercio Electrónico". Sin embargo, Vietnam parece estar interesado en adoptar la E-cc: Vietnam News Agency, Vietnam urged to join e-communications convention, I I de abril de $20 \mathrm{I} 4$, disponible en: http://mutrap. org.vn/index.php/en/newss/mutraps-news/267-vietnam-urged-to-join-e-communicationsconvention 
Aun cuando disposiciones sustantivas de la E-cc son importadas a la legislación modelo regional, no se realiza al mismo tiempo un llamamiento a la adopción formal del tratado.

Con respecto a la relación entre la E-CC y los acuerdos de libre comercio bilaterales y regionales, hay que señalar que son pocos los acuerdos comerciales celebrados en el marco de un proceso de integración económica regional que proporcionen también reglas favorables para los intercambios comerciales, como en el caso de la Unión Europea. Sin embargo, ante la falta de uniformidad en el derecho comercial, los comerciantes pueden enfrentar obstáculos prácticos. Por lo tanto, resulta necesario adoptar textos uniformes que complementen los acuerdos de libre comercio con legislación comercial moderna y eficiente.

Más aún, como ya se ha mencionado, una serie de acuerdos de libre comercio contienen normas relativas a la promoción del uso de las comunicaciones electrónicas mediante la adopción de los principios de no discriminación y de neutralidad tecnológica. Esas reglas a veces exigen específicamente el mantenimiento de la legislación nacional basada en textos de la CNUDMI para asegurar uniformidad legislativa ${ }^{45}$. Disposiciones más específicas sobre el reconocimiento transfronterizo de firmas electrónicas son también comunes ${ }^{4}$. Una manera obvia de implementar dichas disposiciones es a través de la adopción de la E-cC.

La relación entre la facilitación del comercio sin papel y la adopción de la E-CC se hace evidente en el proyecto de texto de un acuerdo regional para la facilitación del comercio transfronterizo sin papel47, el cual está siendo negociando en la Comisión Económica y Social de las Naciones Unidas para Asia y el Pacífico (CESPap). Ese proyecto de acuerdo tiene por objeto establecer un marco jurídico para la armonización regional de los requisitos legales y técnicos relacionados con la facilitación del comercio sin papel. Asimismo, se prevé un mecanismo de aplicación para facilitar la prestación de asistencia técnica a los Estados. El proyecto de acuerdo resalta el hecho de que los textos de la CNUDMI sobre comercio electrónico son las únicas normas legales globales disponibles para construir el marco jurídico necesario para apoyar el comercio sin papel, y también que ya han sido ampliamente adoptados en Asia y el Pacífico. Por lo tanto, se hace hincapié en la importancia de establecer el marco jurídico propicio necesario como cuestión prioritaria, y la conveniencia de hacerlo mediante la adopción del instrumento que garantiza el máximo nivel de uniformidad y previsibilidad jurídica, es decir, la E-cc.

Véase el Acuerdo estableciendo el Área de Libre Comercio asEan-Australia-Nueva Zelanda (AANZFTA), 2009, cap. IO, art. 4.

46 Véase aAnzfta, cap. io, art. 5.

47 Documentos relativos al proyecto de acuerdo se encuentran disponibles en: http://unnext. unescap.org/reso683.asp 
Los marcos contractuales pueden no ser suficientes para asegurar la exigibilidad de los acuerdos en forma electrónica en caso de controversia. En este sentido, el comercio sin soporte en papel se ha incrementado, y los contratos relacionados con la facilitación del comercio también han aumentado el soporte electrónico. Sin embargo, en caso de controversia los acuerdos contractuales en forma electrónica pueden no ser reconocidos por jueces locales, especialmente cuando los contratos se celebraron con partes que tuvieron un limitado contacto previo con los proveedores de servicios de facilitación de comercio. El asunto puede ser resuelto mediante una adopción amplia por parte de los Estados de la E-CC y la inclusión explícita de la E-CC en el contenido contractual.

\section{Conclusiones}

La E-CC ofrece un nuevo estándar para la legislación del comercio electrónico a nivel mundial. Anteriores textos de la CNUDMI sobre comercio electrónico han sido particularmente exitosos en proveer un modelo para la legislación nacional. La E-Cc debe lograr un similar, si no mayor, nivel de aceptación. De hecho, la importancia del comercio electrónico transfronterizo para la economía mundial, reconocido, por ejemplo, también por la ocDE ${ }^{4}$, debe alentar a los Estados a aumentar el ritmo de adopción de la E-cc.

Uno de los límites en el enfoque actual para el estudio y la promoción de la E-CC reside en el hecho de que su relación con otros tratados no está suficientemente explorada. La difusión de más información sobre la E-Cc entre los especialistas de arbitraje parece especialmente importante. De hecho, un número creciente de jurisdicciones apoya el uso de mecanismos alternativos de resolución de conflictos y se ha comprometido a proporcionar el más moderno entorno legislativo. Sin embargo, y a pesar de la creciente utilización de medios electrónicos para celebrar acuerdos arbitrales y en el procedimiento arbitral, no hay una fuerte demanda de la aprobación de la E-CC por parte de la comunidad del arbitraje. Esto es infortunado, teniendo en cuenta los claros beneficios que la adopción de la E-cc traería, por ejemplo, con respecto a los mecanismos de aplicación establecidos en la Convención de Nueva York.

Las jurisdicciones con más experiencia en el uso de las comunicaciones electrónicas y en tratar con cuestiones jurídicas relacionadas deben considerar la adopción de la E-Cc con prontitud. En muchos casos, incluido el de Colombia, esto seguiría una tradición significativa en la adopción temprana de los textos uniformes. Esto también ayudaría a revertir la tendencia actual que se ve en ciertos países de adoptar disposiciones sustantivas de la E-CC solo en la legislación nacional, perdiendo así los beneficios derivados de la adopción formal del tratado.

48 OECD, "Electronic and Mobile Commerce", OECD Digital Economy Papers, n. ${ }^{\circ} 228$, oecd Publishing, 2013 , p. 26, disponible en: http://dx.doi.org/ro.1 $787 / 5 \mathrm{k} 437 \mathrm{p} 2 \mathrm{gxw} 6 \mathrm{~g}$-en 


\section{Bibliografía}

Boss, A. y W. Kilian (eds.), The United Nations Convention on the Use of Electronic Communications in International Contracts: An in-depth guide and sourcebook, 2008.

Butler, P., "The cisg and the United Nations Convention on the Use of Electronic Communications in International Contracts", en I. Schwenzer y L. Spagnolo (eds.), State of Play, Eleven International Publishing, Den Haag, 2013 , pp. I-I 6.

Chong, K. W. y J. S. Chao, "United Nations Convention on the Use of Electronic Communications in International Contracts: A new global standard", Singapore Academy of Law fournal, i 8, pp. I 16-202, 2006 (disponible en línea en http://www.sal.org.sg/digitallibrary/Lists/SAL\% 20Journal/ Attachments/390/2006-i 8-SAcLJ- I I 6-Chong.pdf).

Colección de Tratados de las Naciones Unidas, n. ${ }^{\circ}$ de registro I-50525 (publicación pendiente).

Eiselen, S., "The unecic: International trade in the digital era", Potchefstroom Electronic Law Fournal, 2, pp. I-49, 2007 (disponible en: http://www.puk. ac.za/opencms/export/PUK/html/fakulteite/regte/per/issuepages/2007vo lume Iono2/2007x2x_Eiselen_art.pdf).

Elsan, M. y M. Subaty, "Contract formation using automated message system: survey of Islamic contract law", Arab Law Quarterly, vol. 23, n. ${ }^{\circ}$ 2, pp. I67I $80,2009$.

Feldstein de Cárdenas, S. L. y L. B. Scotti, "La Convención sobre Utilización de las Comunicaciones Electrónicas en los Contratos Internacionales: un avance hacia la armonización legislativa en materia de contratación electrónica", Revista Cientifica de UCES, vol. I I, n. ${ }^{\circ}$ I, pp. 59-89, 2007.

Ferrari, F., "Homeward Trend and Lex Forism Despite Uniform Sales Law", I 3 Vindobona fournal of International Commercial Law \& Arbitration I/2009, pp. I 5-42.

Gabriel, H. D., "The United Nations Convention on the Use of Electronic Communications in International Contracts: An overview and analysis", Uniform Law Review / Revue de droit uniforme, vol. I I, n. ${ }^{2}$, pp. 285-304, 2006.

Нетtenbach, D., Das Übereinkommen der Vereinten Nationen über die Verwendung elektronischer Mitteilungen bei internationalen Verträgen, 2008.

Madrid Parra, A., "El Convenio de Naciones Unidas sobre Contratación Electrónica”, en A. Madrid Parra (dir.), M. J. Guerrero Lebrón (coord.), Derecho patrimonial y tecnología, 2007, pp. 39-I I 3 .

Naciones Unidas, Nota explicativa de la Secretaría de la CNUDMI sobre la Convención de las Naciones Unidas sobre la Utilización de las Comunicaciones Electrónicas en 
los Contratos Internacionales, Nueva York, 2007 (publicación de las Naciones Unidas, n. ${ }^{\circ}$ de venta E.o7.V.2).

oEcD, "Electronic and Mobile Commerce", OECD Digital Economy Papers, n. ${ }^{\circ} 228$, oECD Publishing, 2013 , p. 26, disponible en: http://dx.doi. org/ro. $1787 / 5 \mathrm{k} 437 \mathrm{p} 2$ gxw6g-en

Oviedo Albán, J., "Convención de las Naciones Unidas sobre la Utilización de Comunicaciones Electrónicas en Contratos Internacionales", International Law. Revista Colombiana de Derecho Internacional, vol. 7, pp. 9-57, 2006, disponible en: http://revistainternationallaw.javeriana.edu.co/descargas. php? archivo=rev7_cap. pdf\&idArt $=92 \&$ edicion $=7$

Spagnolo, L., CISG Exclusion and Legal Efficiency, 2014.

uncitral, Promoting confidence in electronic commerce: Legal issues on international use of electronic authentication and signature methods, Viena, 2009 (publicación de las Naciones Unidas, n. ${ }^{\circ}$ de venta E.o9.V.4).

UNCTAD, Review of e-commerce legislation harmonization in the Association of Southeast Asian Nations, Nueva York y Ginebra, 2013, United Nations Doc. Unctad/ DTL/STICT/2OI3/I.

\section{Legislación}

Acuerdo del Área de Libre Comercio ASEAN-Australia-Nueva Zelanda (AANZFTA), 2009 .

Conferencia de Derecho Uniforme de Canadá, Sección de Derecho Civil, Ley Uniforme de Comunicaciones Electrónicas (Uniform Law Conference of Canada, Civil Law Section, Uniform Electronic Communications Convention Act) aprobada en Winnipeg, Manitoba, agosto de 20 I I.

Conferencia de Derecho Uniforme de Canadá, Sección de Derecho Civil, Ley Uniforme de Comunicaciones Electrónicas (Uniform Law Conference of Canada, Civil Law Section, Uniform Electronic Communications Convention Act) aprobada en Winnipeg, Manitoba, agosto de 20 I I.

Convención sobre el Reconocimiento y la Ejecución de las Sentencias Arbitrales Extranjeras, hecha en Nueva York el ro de junio de $195^{8}$, adoptada por la CNUDMr el 7 de julio de 2006 en su trigésimo noveno período de sesiones (Naciones Unidas Doc. A / 6I/I 7, anexo II).

Convención sobre el Reconocimiento y la Ejecución de las Sentencias Arbitrales Extranjeras, hecha en Nueva York el ro de junio de $195^{8}$, adoptada por la CNUDMI el 7 de julio de 2006 en su trigésimo noveno período de sesiones (Naciones Unidas Doc. A/6I/I 7 , anexo II).

Decreto 57/2006/ND-cP del 9 de junio de 2006, "Decreto sobre Comercio Electrónico", Vietnam. 
Ley 527 de r999 de Colombia.

Ley de Transacciones Electrónicas (2008) de Omán.

Ley Modelo de comesa en Transacciones Electrónicas y Guía para la incorporación de 20 Io (COMESA Model Law on Electronic Transactions and Guide to Enactment 20IO).

Ley Modelo de la cNudmi sobre Comercio Electrónico con la Guía para su incorporación al derecho interno, junto con su nuevo artículo 5 bis aprobado en I998, Nueva York, I 999 (publicación de las Naciones Unidas, n. ${ }^{\circ}$ de venta S.99.V.4).

Ley Modelo de la cNudmi sobre las Firmas Electrónicas con la Guía para su incorporación al derecho interno, Nueva York 2002 (publicación de las Naciones Unidas, n. ${ }^{\circ}$ de venta S.02.V.8).

Regulación (EU) n. ${ }^{\circ}$ 9ro/20r4 del Parlamento Europeo y del Consejo sobre servicios de identificación y confianza electrónicos para las transacciones electrónicas en el mercado interior (eIDAs).

Vietnam News Agency, Vietnam urged to join e-communications convention, I I de abril de 20I4, disponible en: http://mutrap.org.vn/index.php/en/newss/ mutraps-news $/ 267$-vietnam-urged-to-join-e-communications-convention

\section{Jurisprudencia}

Corte Laboral de Sudáfrica (Durban), Caso n. ${ }^{\circ} \mathrm{D}_{204 / 07}$ (г. ${ }^{\circ}$ de julio de 2008) Jafta v. Ezemvelo KZN Wildlife [2008] zALC 84; [2008] Iо BLLR 954 (LC); (2009) 30 ILJ I 3 I (LC); Caso Clout, Case 964, disponible en: http://www.saflii.org/ $\mathrm{za} / \mathrm{cases} / \mathrm{zALC} / 2008 / 84 \cdot \mathrm{html}$

Corte Suprema de Singapur, SM Integrated Transware Pte Ltd. v. Schenker Singapore (Pte) Ltd., n. 594 de 2003, [2005] sGHC 58, Caso clout C 66r .

Cour d'appel du Québec (Canada), Services financiers Paccar ltée c. Kingsway, Compagnie d'assurances générales, 3 I de mayo de 20I2; 2012 QCCA IO3O (CanLII); Caso Clout i I95, disponible en: http://canlii.ca/t/frm9m 